\title{
JOINT DELAY AND AZIMUTH ESTIMATION OF COHERENT WAVES FOR MILLIMETER-WAVE BAND CHANNEL SOUNDING
}

\author{
Kentaro Saito $^{1}$, Ahmad Salaam Mirfananda', Jun-ichi Takada1, \\ Mitsuki Nakamura ${ }^{2}$, Wataru Yamada ${ }^{2}$, and Yasushi Takatori ${ }^{2}$ \\ ${ }^{1}$ School of Environment and Society, Tokyo Institute of Technology, Tokyo, Japan, Tel: +81 357342551 , \\ e-mail: saitouken@tse.ens.titech.ac.jp, takada@tse.ens.titech.ac.jp, mirfananda.a.aa@m.titech.ac.jp \\ ${ }^{2}$ NTT Access Network Service System Laboratories, NTT Corporation, Kanagawa, Japan, \\ e-mail: mitsuki.nakamura.rb@hco.ntt.co.jp, wataru.yamada.es@hco.ntt.co.jp, \\ yasushi.takatori.rk@hco.ntt.co.jp
}

Received Date: April 17, 2019; Revised Date: November 19, 2019; Acceptance Date: November 21, 2019

\begin{abstract}
The user traffic in the mobile communication area has rapidly increased owing to the widespread of smartphones and various cloud services. To handle the increasing traffic, in the fifth-generation mobile communication system (5G), the millimeter-wave multiple-input and multiple-output (MIMO) communication technology is under development. Because the MIMO transmission performance heavily depends on the radio propagation characteristics, various MIMO channel measurements are needed for the performance evaluation and system design. The accurate and efficient parameter estimation algorithm which estimates the propagation delays and angle of arrivals (AoA) of radio waves is also indispensable for the purpose. In this paper, we extended the joint delay and azimuth estimation (JADE) method based on multiple signal classification (MUSIC) algorithm. In our proposal, the drawback of the MUSIC that the performance degrades for the estimation of coherent waves was solved by applying the smoothing technique in the frequency domain. It also makes the antenna calibration simpler. We implemented the proposed algorithm for the channel sounding system in the $66 \mathrm{GHz}$ band, which is one of the candidate frequency bands for the International Mobile Telecommunications (IMT) system and evaluated the effectiveness through the experiment in an anechoic chamber. The result showed that our proposed method can de-correlate the signal components of coherent waves, and improved the parameter estimation accuracy significantly. The root means square error (RMSE) of the propagation delay estimation was improved from $2.7 \mathrm{~ns}$ to $0.9 \mathrm{~ns}$, and the RMSE of the AoA estimation was improved from $20.3 \mathrm{deg}$. to 7.2 deg. The results are expected to be utilized for the millimeter wave band MIMO channel modeling.
\end{abstract}

Keywords: Channel sounding, Coherent wave, Joint delay and angular estimation, Millimeterwave wireless communication, Propagation parameter estimation, Smoothing technique

\section{Introduction}

The user traffic in the mobile communication area has rapidly increased owing to the widespread of smartphones and various cloud services. Therefore, in the fifth-generation mobile communication system $(5 \mathrm{G})$, the millimeter-wave wireless communication is expected to play an important role to improve the communication capacity [1]. in the higher frequency band, it is known that the reflection and diffraction losses become significant compared to the conventional band. Since the multiple-input and multiple-output (MIMO) transmission performance heavily depends on the propagation characteristics, MIMO channel models were developed for the performance evaluation and design of the system [2-4]. In those models, the 
propagation channel is represented by the superposition of numerous radio waves which are defined by model parameters such as propagation delays and angle of arrivals (AoA). Because the distributions of those propagation parameters change significantly according to frequency bands and communication scenarios, the exhaustive channel measurements are needed. And, the efficient and accurate parameter estimation method from the measurements is also indispensable.

In this paper, we proposed the novel propagation parameter estimation method based on the multiple signal classification (MUSIC) algorithm [5] for the purpose. Although the MUSIC algorithm has the advantage of fast calculation, it also has the disadvantage that the algorithm does not work if the incident waves are coherent. Although the spatial smoothing technique was proposed to solve the issue [6], the method requires that the array response has the Vandermonde structure, and this assumption is not feasible practically if the array antenna has other structures or the antenna radiation pattern is not isotropic. In our proposal, we extended the MUSIC power spectrum calculation to both the frequency and AoA domains based on the Joint Angle and Delay Estimation (JADE) MUSIC algorithm [7]. The smoothing technique was applied to the frequency domain to de-correlate the coherent incident waves. Because any additional calculations such as beam-space transformation [8] are not needed in the AoA domain, our proposal has the advantage to make the antenna calibration to be simpler. Another contribution of the paper is that we implemented the proposed parameter estimation method for the channel sounding system [9] in $66 \mathrm{GHz}$ band, which is one of the candidate frequency bands for the future development of International Mobile Telecommunications (IMT) system in World Radiocommunication Conference (WRC)19. And, we showed the effectiveness of our proposal through the actual measurement. The estimation performance was evaluated in the anechoic chamber. The result showed that our proposal improved the estimation accuracies of delays and AoAs of two close incident waves.

\section{Joint Delay and Azimuth Estimation Algorithm for Coherent Waves}

The overview of our proposed estimation method is shown in Figure 1. In this paper, we consider the uniform circular array (UCA). The array consists of $M$ antenna elements that locate at $\Delta \eta$ interval. We also assume that the wideband orthogonal frequency division multiplexing (OFDM) signal was measured by each antenna. Here, $\Delta f$ and $N$ are the sub-carrier spacing and sub-carrier number of the signal. Therefore, the measured data $\boldsymbol{x} \in \mathbb{C}^{N \times M}$ is obtained from the measurement.

\section{JADE-MUSIC Algorithm}

In the JADE-MUSIC algorithm [7], the propagation delay $\tau$ and AoA $\phi$ of incident wave are jointly estimated from the MUSIC power spectrum. The signal model is defined by the joint delay and AoA domain array response $\boldsymbol{\alpha}(\tau, \phi)$ that consists of the AoA domain array response $\boldsymbol{\alpha}_{\mathrm{AoA}}(\phi)$ and the frequency domain array response $\boldsymbol{\alpha}_{\mathrm{F}}(\tau)$.

$$
\begin{aligned}
& \boldsymbol{\alpha}_{\mathrm{AoA}}(\phi)=\left[\exp \left(\frac{j 2 \pi}{\lambda} r \cos (\phi)\right), \exp \left(\frac{j 2 \pi}{\lambda} r \cos (\phi-\Delta \eta)\right), \cdots\right. \\
& \left.\quad \exp \left(\frac{j 2 \pi}{\lambda} r \cos (\phi-(M-1) \Delta \eta)\right)\right] \\
& \boldsymbol{\alpha}_{\mathrm{F}}(\tau)=[1, \exp (-j 2 \pi \Delta f \tau), \cdots \exp (-j 2 \pi(N-1) \Delta f \tau)]
\end{aligned}
$$




$$
\boldsymbol{\alpha}(\tau, \phi)=\boldsymbol{\alpha}_{\mathrm{F}}(\tau) \otimes \boldsymbol{\alpha}_{\mathrm{AoA}}(\phi)
$$

Here, $n(1 \leq n \leq N)$ is the sub-carrier index of the signal, $m(1 \leq m \leq M)$ is the antenna element index, and $r$ is the radius of UCA. $\lambda$ is the wavelength of the carrier wave, and $\otimes$ represents the Kronecker product. The power spectrum of JADE-MUSIC is calculated as same as the conventional MUSIC algorithm.

$$
P(\tau, \phi)=\frac{\boldsymbol{\alpha}(\tau, \phi)^{H} \boldsymbol{\alpha}(\tau, \phi)}{\boldsymbol{\alpha}(\tau, \phi)^{H} \boldsymbol{E}_{N} \boldsymbol{E}_{N}^{H} \boldsymbol{\alpha}(\tau, \phi)}
$$

Here, $\boldsymbol{E}_{\mathrm{N}}$ is the noise subspace that is obtained by the singular value decomposition (SVD) of measured data $\boldsymbol{x}$ [5]. The propagation delays and AoAs of the incident waves are estimated by the peak search of the spectrum.

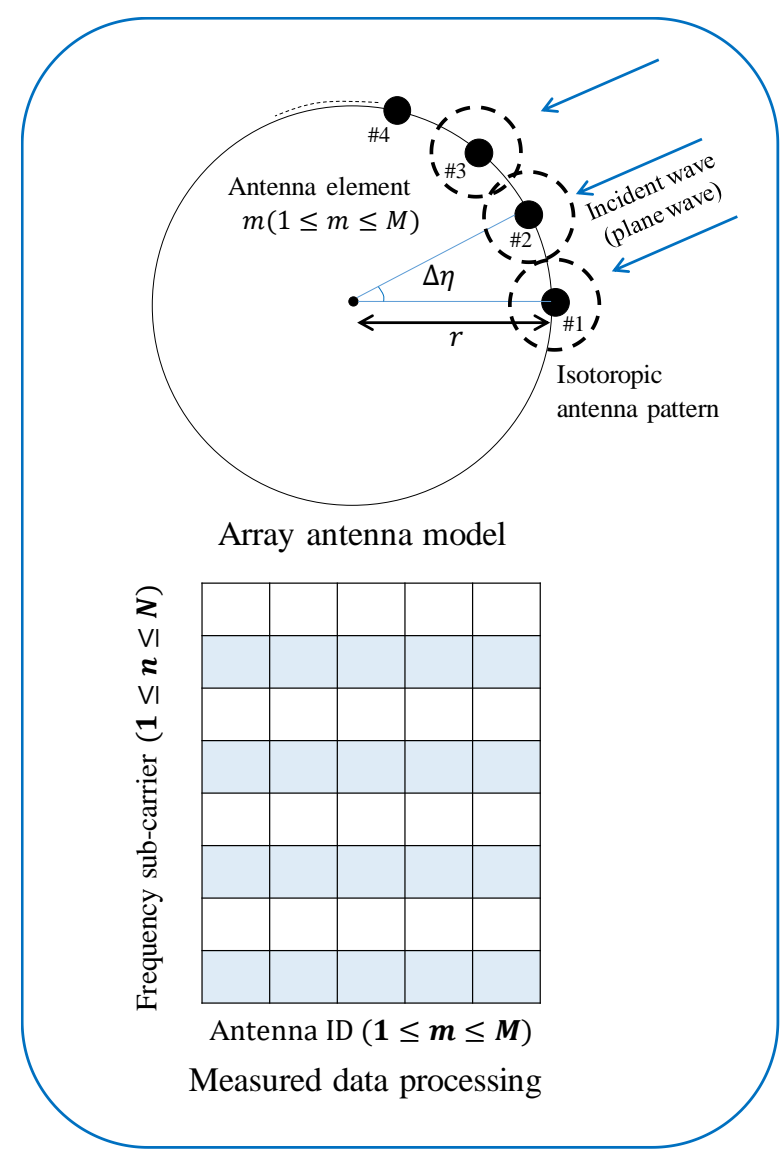

(a) JADE MUSIC

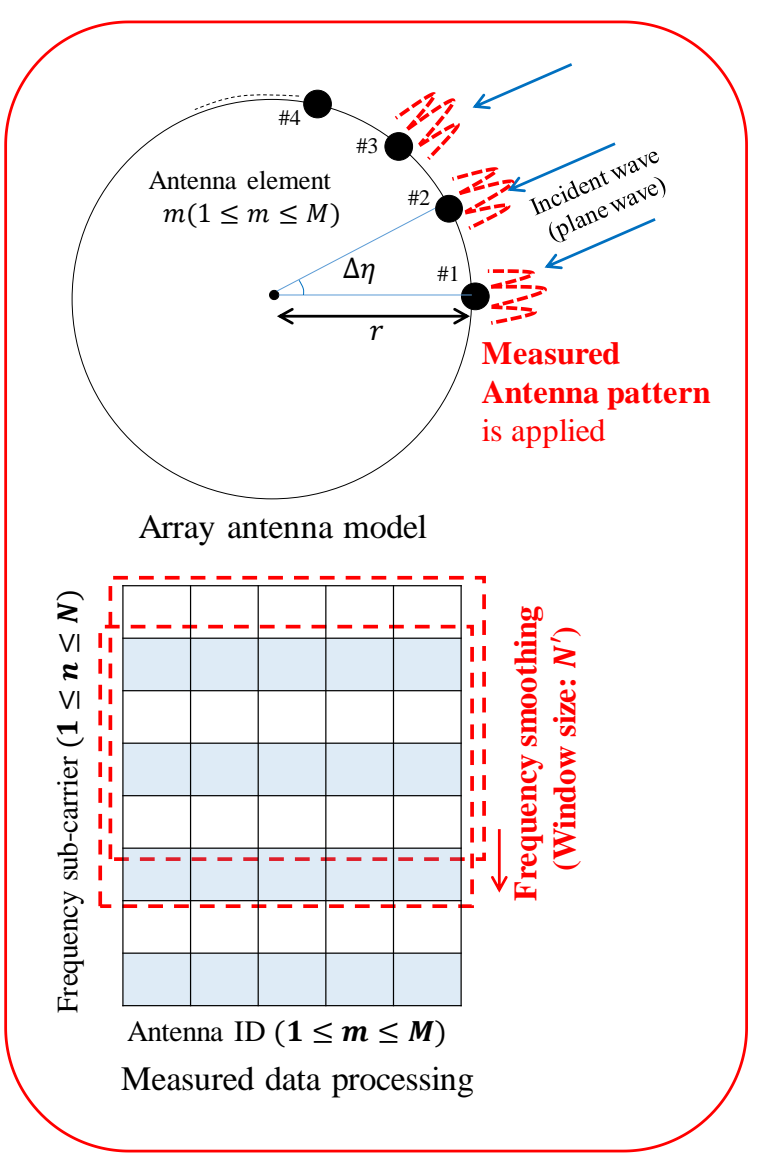

(b) Proposed estimation method

Figure 1. The overview of the proposed estimation method

\section{Frequency Smoothing (FS) Technique and Antenna Radiation Pattern Consideration}

It is known that the MUSIC algorithm has the drawback that propagation parameters are not estimated correctly if the incident waves are coherent. This is because each incident wave is not separately identified in the signal subspace calculated by the SVD. Therefore, to de-correlate the signal components of incident waves, we propose to apply the smoothing technique to the measured data $\boldsymbol{x}$ in the frequency domain. The smoothing calculations are as follows. 


$$
\boldsymbol{x}^{\prime}=\boldsymbol{W} \boldsymbol{x}
$$

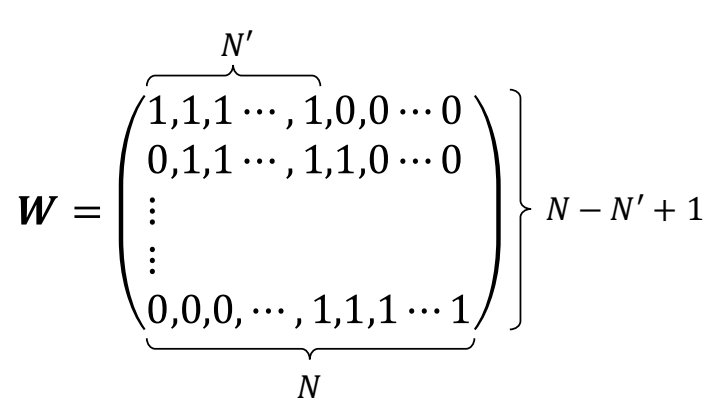

The smoothing is the same as the moving average calculation, and $N^{\prime}$ corresponds to the window size. The noise subspace $\boldsymbol{E}_{\mathrm{N}}{ }^{\prime}$ is calculated from the smoothed data $\boldsymbol{x}^{\prime}$.

Next, we explain the antenna radiation pattern consideration. Because the theoretical AoA domain array response shown in (1) assumes the isotropic antenna, it can cause the estimation error if the actual antenna radiation pattern is different from the isotropic pattern. Therefore, we applied the antenna radiation pattern which was obtained from another measurement to the AoA domain array response calculation for the calibration purpose.

$$
\alpha_{\mathrm{AoA}}{ }^{\prime}(\phi)=g(\phi) \circ \alpha_{A o A}(\phi)
$$

Here, $\boldsymbol{g}(\phi)$ is the antenna pattern of each antenna element and $\circ$ is the Hadamard product. The modified array response is defined as $\boldsymbol{\alpha}^{\prime}(\tau, \phi)=\boldsymbol{\alpha}_{\mathrm{F}}{ }^{\prime}(\tau) \otimes \boldsymbol{\alpha}_{\mathrm{AoA}}{ }^{\prime}(\phi) . \boldsymbol{\alpha}_{\mathrm{F}}{ }^{\prime}(\tau)=$ $\left[\alpha_{\mathrm{F}, 1}(\tau), \alpha_{\mathrm{F}, 2}(\tau), \cdots \alpha_{\mathrm{F}, \mathrm{N}}(\tau)\right]$ by taking the change of subcarrier number by smoothing processing into consideration. Finally, the modified MUSIC power spectrum $P^{\prime}(\tau, \phi)$ is calculated as follows.

$$
P^{\prime}(\tau, \phi)=\frac{\alpha(\tau, \phi)^{\mathrm{H}} \alpha^{\prime}(\tau, \phi)}{\alpha^{\prime}(\tau, \phi)^{\mathrm{H}}{E_{\mathrm{N}}}^{\prime}{E_{\mathrm{N}}}^{{ }^{\mathrm{H}}} \alpha^{\prime}(\tau, \phi)}
$$

The advantage of our proposal is to combine the smoothing calculation and the antenna calibration which are indispensable to improve the estimation accuracy for the real measurement system.

\section{Experiment in Anechoic Chamber}

We conducted the experiment in an anechoic chamber for the performance evaluation of the proposed algorithm. The measurement layout is shown in Figure 2, and the measurement parameters are summarized in Table 1 . The transmitter $(\mathrm{Tx})$ and the receiver $(\mathrm{Rx})$ were fixed in the anechoic chamber. The distance between the Tx antenna and the Rx antenna was 6.8 $\mathrm{m}$. If the $\mathrm{Tx}$ and $\mathrm{Rx}$ antenna heights are different, the elevation AoA estimation is also necessary. To simplify the experiment condition, both antenna heights were set to $2.1 \mathrm{~m}$ by using the positioning laser equipment. This height was also high enough to prevent the obstruction of radio waves by the absorbers. A steal reflector was set at the points from B1 to B7 to make the two coherent incident wave condition with different incident angle conditions. The size of the reflector was $90 \mathrm{~cm}$ height by $150 \mathrm{~cm}$ width, and it can be regarded as an ideal plane surface. The setting angle of the reflector was adjusted to satisfy the reflection law at each point. 


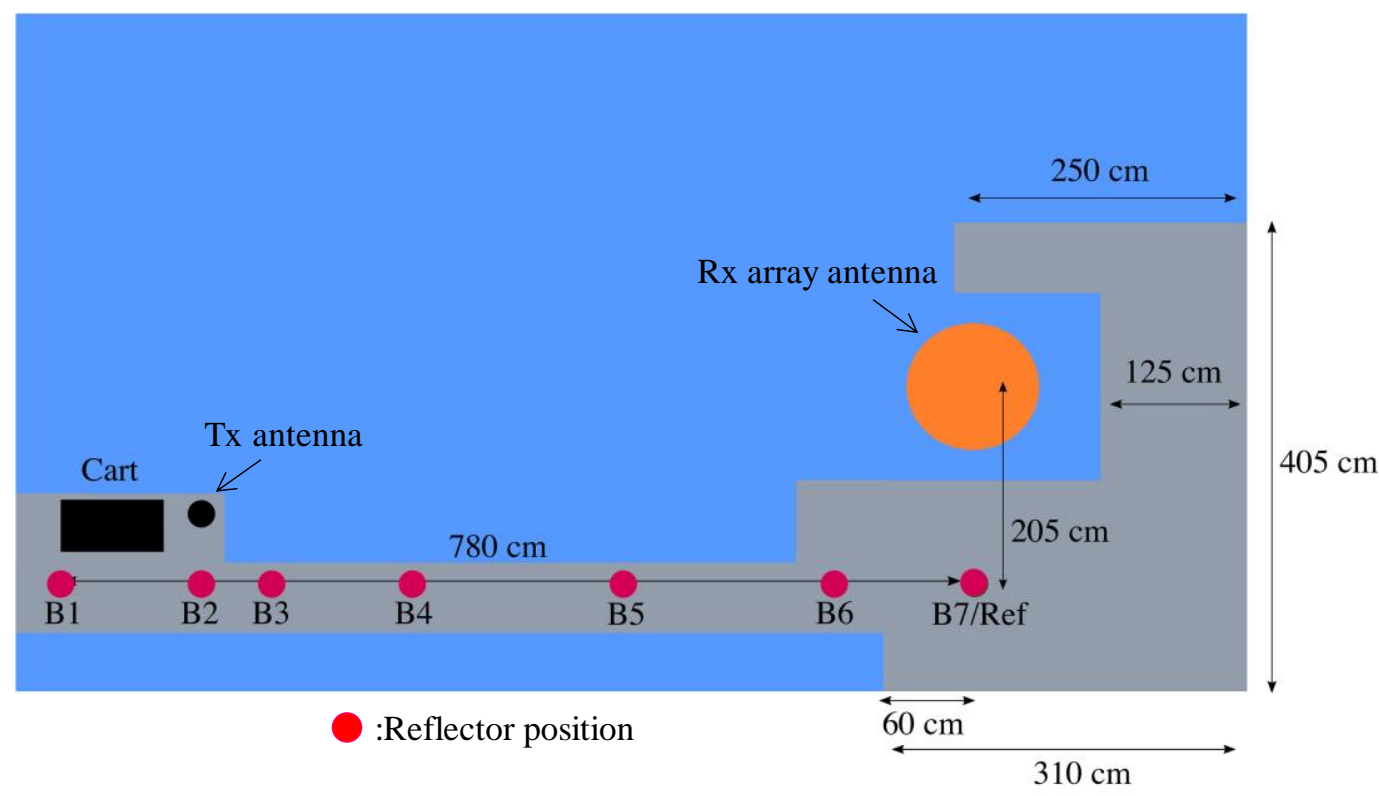

Figure 2 Measurement layout

Table 1. Measurement Parameters

\begin{tabular}{|c|c|}
\hline \multicolumn{2}{|c|}{ Measurement Setting } \\
\hline Center frequency & $66.5 \mathrm{GHz}$ \\
\hline Signal bandwidth & $500 \mathrm{MHz}$ \\
\hline Transmission power & $10 \mathrm{dBm}$ \\
\hline Sub-carrier num. & $\mathrm{N}=200$ \\
\hline Tx/Rx Antennas & Monopole antenna $(2 \mathrm{dBi})$ \\
\hline Rx array antenna & $\begin{array}{l}\text { Virtual UCA ( } \mathrm{r}=0.049 \mathrm{~m} \text {, } \\
\qquad \mathrm{M}=200)\end{array}$ \\
\hline Antenna height & $2.1 \mathrm{~m}$ \\
\hline \multicolumn{2}{|c|}{ Estimation Setting } \\
\hline Number of waves & 2 \\
\hline Smoothing Window Size & $N^{\prime}=150$ \\
\hline
\end{tabular}

The photos and block diagram of the channel sounder and the reflector are shown in Figure 3. The OFDM signal was generated by the signal generator R\&S SMR40 and transmitted from the monopole antenna. The Rx monopole antenna was fixed on the rotator, and the rotator was controlled by the personal computer (PC). The Rx antenna rotated circularly on the rotator, and it formed the virtual UCA. The virtual array method has the advantage to simplify the antenna calibration procedure. The received signal was recorded by the digital oscilloscope R\&S RTO2044. The Cesium clock was connected to both equipment to achieve the synchronization. The center frequency was $66.5 \mathrm{GHz}$, and the signal bandwidth was $500 \mathrm{MHz}$. Detailed system information is introduced in [9]. The angular characteristics of waves can be obtained by the method described in the previous section. We compared the parameter estimation performance between the conventional JADE-MUSIC algorithm and our proposed algorithm. The window size selection is the tradeoff between the de-correlation performance of coherent waves and the delay estimation resolution. In this paper, the window size $\mathrm{N}^{\prime}$ was 150 to suppress the excessive degradation of delay resolution. The antenna pattern for the calibration was also measured in the same measurement condition without the reflector. 


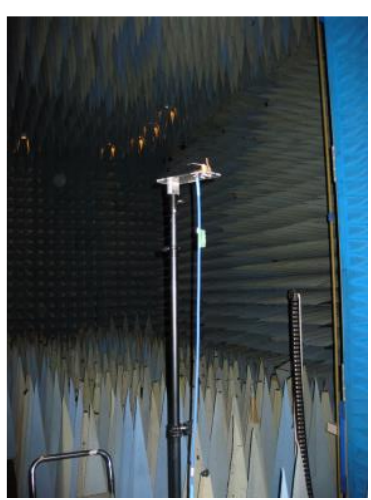

(a) Tx antenna

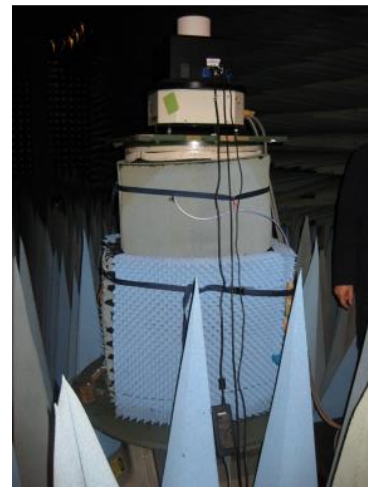

(b) Rx array antenna

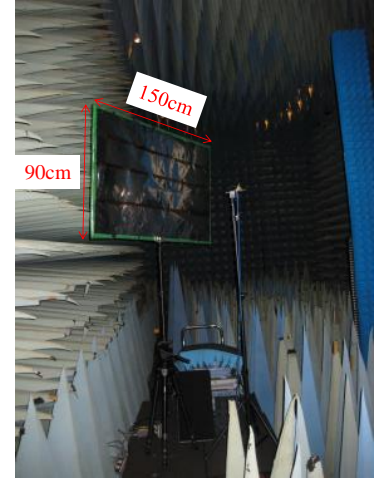

(c) Reflector

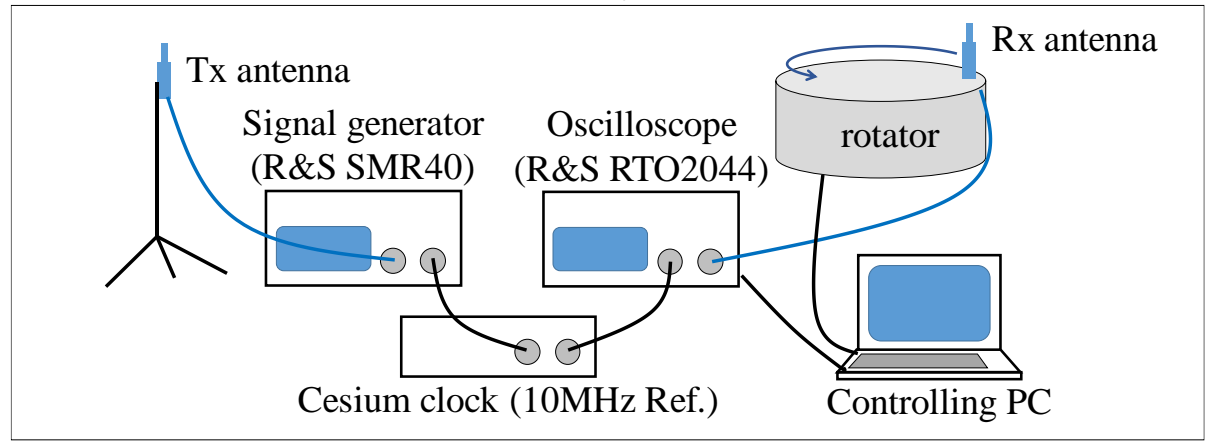

(d) Block diagram of the system

Figure 3. Measurement system ((a)Tx antenna, (b)Rx array antenna, (c) Reflector, (d) Block diagram of the system)

\section{Measurement Results}

\section{MUSIC Power Spectrum Comparison}

The power spectra of JADE-MUSIC and the proposed method when the reflector was set at B1 are shown in Figure 4. In the power spectra of JADE-MUSIC, although the peak of direct wave is observed, the spectrum was distorted, and the peak level was low. The peak of the reflection wave was not distinguishable from the noise floor. On the contrary, in the proposed method, the peaks became much clear, and both two incident waves were identified from the spectrum. The result proved the effectiveness of the proposed method for the parameter estimation of coherent waves in the real measurement system.

\section{Propagation Delay and AoA Estimation Accuracy}

The propagation delay and AoA estimation results of two incident waves are summarized in Figure 5. In the figure, the theoretical values which were calculated from the measurement setting are also shown. About the propagation delay, the delay difference between the direct wave and reflection wave was minimized when the reflector position was B4. In contrast, the angle difference was minimized at B1, and it monotonically increased as the reflector position changed. The estimation becomes more difficult when those differences are small. In JADE-MUSIC, two incident waves were separately identified only in B5 and B6 cases. The result means that the two waves were separately identified only when both the delay and AoA differences were significant. On the contrary, in the proposed method, the two waves were separately identified also in B1 and B2 cases. This is because the signal coherence between two waves was successfully resolved by the smoothing processing as explained in the previous section. The proposed method also failed to detect the reflection wave in B5 
case because the sidelobe of the direct wave is mis-detected as the second wave. Although it is not practical to control the sidelobe characteristics of the spectrum, it might be possible to switch the estimation algorithm if the clear peak is not detected by a single algorithm. The root means square error (RMSE) between the estimation results and the theoretical value is summarized in Table 2. The RMSE was calculated from both results of the direct and reflection waves at all reflection positions. There is an ambiguity of the matching between the theoretical value and the estimation result because there are two data which correspond to the direct wave and the reflection wave. Therefore, in this paper, we selected the nearest pair which minimizes the RMSE result. The RMSE of AoA was also calculated by the same method. While the RMSEs of the propagation delay and the AoA were $2.7 \mathrm{~ns}$ and $20.3 \mathrm{deg}$. in JADE-MUSIC, those were significantly improved to $0.9 \mathrm{~ns}$ and $7.2 \mathrm{deg}$. in our proposal.

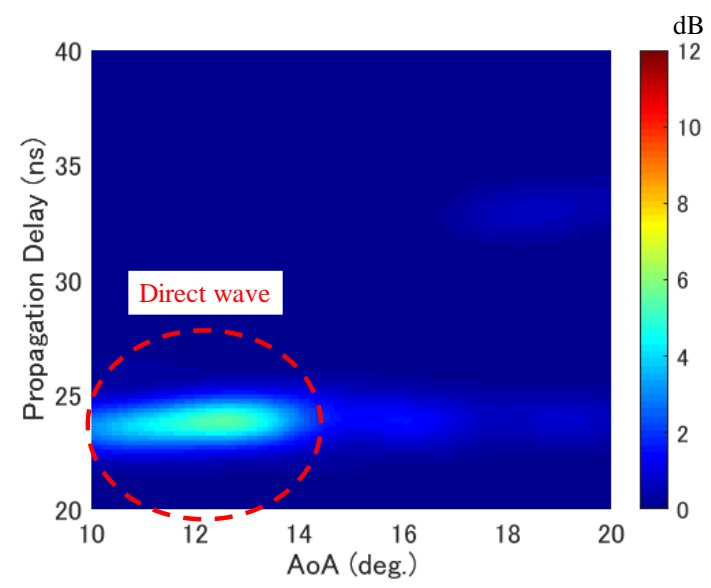

(a) JADE MUSIC

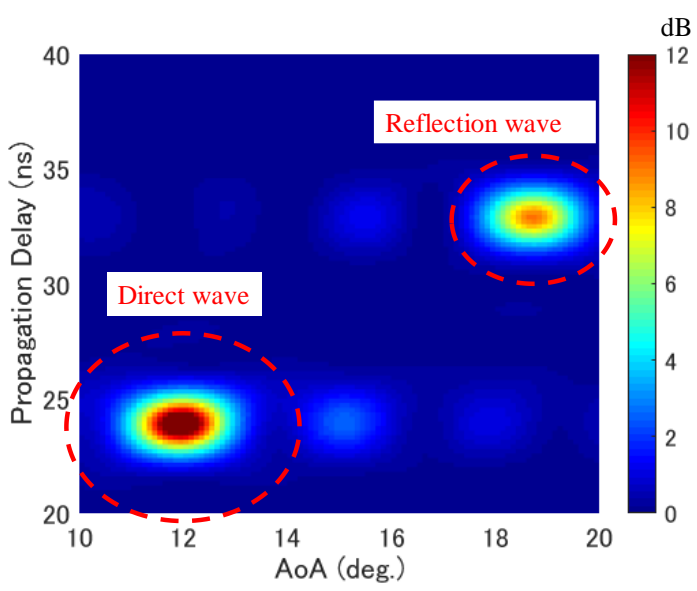

(b) Proposed method

Figure 4. MUSIC Power Spectrum Comparison at B1

((a) JADE-MUSIC, (b) Proposed method)

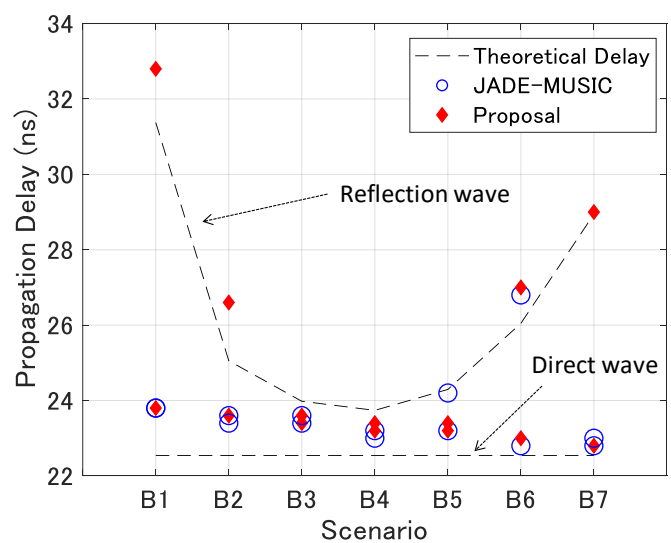

(a) Propagation Delay

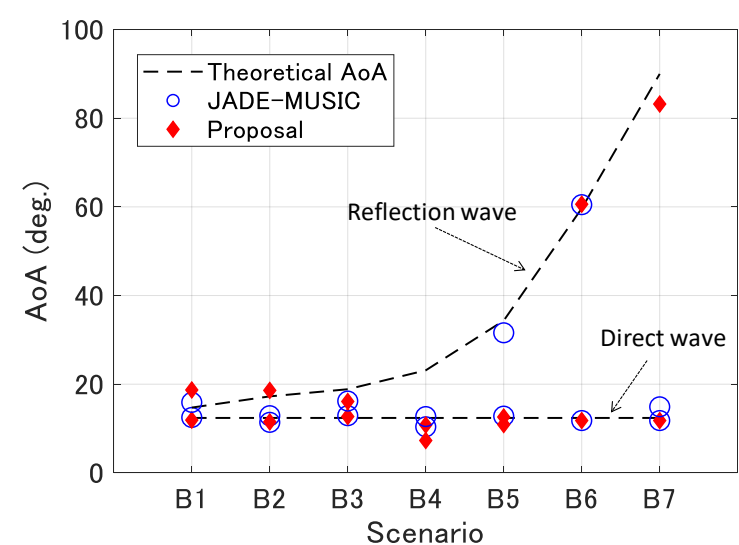

(b) AoA

Figure 5. Propagation delay and AoA estimation result summary

Table 2. RMSE Summary of Parameter Estimation

\begin{tabular}{|c|c|c|}
\hline & JADE MUSIC & Proposed Method \\
\hline Propagation Delay & $2.7 \mathrm{~ns}$ & $0.9 \mathrm{~ns}$ \\
\hline AoA & $20.3 \mathrm{deg}$. & $7.2 \mathrm{deg}$. \\
\hline
\end{tabular}




\section{Conclusions}

In this paper, we extended the JADE-MUSIC algorithm for the parameter estimation of coherent waves in the real measurement system. Although the conventional MUSIC algorithm has the drawback that the estimation accuracy decreases in case of coherent incident waves, our proposal solved the issue by applying the smoothing technique in the frequency domain. It also makes the antenna calibration simpler. We implemented the proposed algorithm for the $66 \mathrm{GHz}$ band channel sounding system and evaluated the effectiveness through the experiment in an anechoic chamber. The result showed that our proposed method made the MUSIC power spectrum much clearer than the conventional JADE-MUSIC algorithm, and it significantly improved the propagation parameter estimation accuracy. The RMSE of propagation delay estimation was improved from $2.7 \mathrm{~ns}$ to $0.9 \mathrm{~ns}$ and the RMSE of AoA estimation was improved from $20.3 \mathrm{deg}$. to $7.2 \mathrm{deg}$. The further experiment in the real environment where many coherent waves arrive will be the future work to clarify the applicability of our proposal. And then, the channel measurements in various propagation environments and parameterizing the MIMO channel are planned. The results are expected to be utilized for the millimeter wave band MIMO channel modeling.

\section{Reference}

[1] T.S. Rappaport, S. Sun, R. Mayzus, H. Zhao, Y. Azar, K. Wang, G.N. Wong, J.K. Schulz, M. Samimi, and F. Gutierrez, "Millimeter wave mobile communications for 5G cellular: It will work!," IEEE Access, Vol. 1, pp. 335-349, 2013.

[2] J. Medbo, P. Kyosti, K. Kusume, L. Raschkowski, K. Haneda, T. Jamsa, V. Nurmela, A. Roivainen, and J. Meinila, "Radio propagation modeling for $5 \mathrm{G}$ mobile and wireless communications," IEEE Communications Magazine, Vol. 54, No. 6, pp. 144-151, 2016.

[3] 3GPP, Technical Specification Group Radio Access Network: Study on Scenarios and Requirements for Next Generation Access Technologies (Release 14), (TR 38.900 v14. 0. 0), 2016 [Online]. Available:

https://portal.3gpp.org/desktopmodules/Specifications/SpecificationDetails.aspx?specif icationId=2991

[4] ITU-R, Guidelines for Evaluation of Radio Interface Technologies for IMT-2020 (WP 5D Draft New Report ITU-R M. [IMT-2020.EVAL]), 2017 [Online]. Available: https://www.itu.int/pub/R-REP-M.2412-2017

[5] R.O. Schmidt, "Multiple emitter location and signal parameter estimation," IEEE Transactions on Antennas and Propagation, Vol. 34, No. 3, pp. 276-280, 1986.

[6] U. Pillai, and B.H. Kwon, "Forward/backward spatial smoothing techniques for coherent signal identification," IEEE Transactions on Acoustics, Speech, and Signal Processing, Vol. 37, No. 1, pp. 8-15, 1989.

[7] M.C. Vanderveen, B.C. Ng, C.B. Papadias, and A. Paulraj, "Joint angle and delay estimation (JADE) for signals in multipath environments," In: Conference Record of the Thirtieth Asilomar Conference on Signals, Systems and Computers, Vol. 2, pp. 12501254, 1996.

[8] C.P. Mathews, and M.D. Zoltowski, "Eigenstructure techniques for 2-D angle estimation with uniform circular arrays," IEEE Transaction on Signal Processing, Vol. 42, No. 9, pp. 2395-2407, 1994.

[9] H. Nguyen, W. Keusgen, and T. Eichler, "Instantaneous direction of arrival measurements in mobile radio channels using virtual circular array antennas," In: Proceedings of 2016 IEEE Globecom Workshops (GC Workshops), pp. 1-7, 2016. 